OPEN ACCESS

Edited by:

Marcelo Horacio Miragaya,

University of Buenos Aires, Argentina

Reviewed by:

Ayman Abdel-Aziz Swelum

Zagazig University, Egypt

Shenming Zeng,

China Agricultural University, China

*Correspondence:

Yuchen Nan

nanyuchen2015@nwsuaf.edu.cn

${ }^{\dagger}$ These authors share first authorship

Specialty section: This article was submitted to Animal Reproduction -

Theriogenology,

a section of the journal

Frontiers in Veterinary Science

Received: 14 November 2021

Accepted: 26 January 2022

Published: 18 February 2022

Citation:

Zhang B, Qu G, Nan Y and Zhou E-M

(2022) Ovarian Oxidative Stress Induced Follicle Depletion After Zona Pellucida 3 Vaccination Is Associated With Subfertility in BALB/C Mice.

Front. Vet. Sci. 9:814827. doi: 10.3389/fvets.2022.814827

\section{Ovarian Oxidative Stress Induced Follicle Depletion After Zona Pellucida 3 Vaccination Is Associated With Subfertility in BALB/c Mice}

\author{
Beibei Zhang ${ }^{1,2 \dagger}$, Guanggang Qu ${ }^{3 \dagger}$, Yuchen Nan ${ }^{1,2 *}$ and En-Min Zhou ${ }^{1,2}$ \\ ${ }^{1}$ Department of Preventive Veterinary Medicine, College of Veterinary Medicine, Northwest A\&F University, Yangling, China, \\ ${ }^{2}$ Scientific Observing and Experimental Station of Veterinary Pharmacology and Diagnostic Technology, Ministry of \\ Agriculture, Yangling, China, ${ }^{3}$ Shandong Binzhou Animal Science and Veterinary Medicine Academy, Binzhou, China
}

Impaired follicular development associated with autoimmune ovarian disease (AOD), is a typical side effect of ZP3 vaccine-induced subfertility and contributes to the fertility decline, but the mechanism is unknown. In this study, a AOD model was established with recombinant mouse zona pellucida 3 (mZP3) protein in the BALB/c mice, and coadministrated with $0.5 \mathrm{mg} / \mathrm{kg}$ antioxidant stress drug sodium selenite (SS), whereas intraperitoneal injection was used and the relationships among oxidant stress (OS), follicle loss and fertility were evaluated. Here we demonstrated that ZP3 vaccination elicited high antibody titers and correlated with reductions of ovarian follicle numbers in both fertile and infertile mice, whereby magnitudes of both factors were negatively correlated with litter size. Moreover, increased OS in ovaries of mZP3-immunized mice was related to high levels of reactive oxygen species (ROS) and malondialdehyde (MDA), and is accompanied by a decrease in the total antioxidant capacity (TAC) of ovaries. Meanwhile, activation of caspase-3 and caspase-9 along with increased Bax and decreased Bcl-2 levels were observed, indicating the ongoing apoptosis of ovarian cells. Notably, inhibition of OS with SS reduced ovarian ROS and apoptosis levels, which was consisted with restoration of follicle numbers. More importantly, SS treatment when co-administered concurrently with mZP3 immunization led to significantly improved fertility $(P<0.05)$ and the average litter size of the mZP3-vaccinated SS-treated group increased by $\sim 29.2 \%$ as compared with that of the vaccinated but untreated group. In conclusion, infertility caused by ZP3 vaccination was mechanistically associated with ovarian OS which triggered depletion of ovarian follicles.

Keywords: zona pellucida 3, subfertility, ovary, follicle depletion, oxidative stress, apoptosis

\section{INTRODUCTION}

Zona pellucida 3 (ZP3), the primary oocyte receptor for sperm recognition, has been recognized as a potential candidate for use as birth controlling target in mammalian species by immunizing using $\mathrm{ZP} 3$ vaccination (1-3). However, ZP3 immunization is associated with AOD and typically induces symptoms of premature ovarian failure $(\mathrm{POF})(4,5)$, thus greatly limiting its applicability as a contraceptive (5). Interestingly, development of ovarian pathology could be blocked after 
disruption of the ZP3 pathogenic epitopes (6) or improved after adoptive transfers of $\mathrm{CD} 4{ }^{+} \mathrm{CD} 25^{+}$Tregs $(4,7)$. Although individual differences are observed among experimental subjects, ZP3-induced ovarian inflammation is closely associated with ovarian dysfunction and fertility, with severe inflammation often accompanied with fewer follicles and smaller litter sizes $(4,8)$. Of note, vaccination-induced ovarian dysfunction is characterized by follicular atresia, vacuolation, luteinization and other signs that may be responsible for the reduction or total elimination of follicles and subsequent infertility $(9,10)$. Therefore, ovarian inflammation may be related to these pathological effects despite its role in fertility decline remains unclear.

Reactive oxygen species (ROS), a by-product of mitochondrial processes associated with energy metabolism, participates in the regulation of follicle maturation during different developmental stages when presents at physiological concentrations (11). However, abnormal persistence of ROS in tissues, especially during chronic inflammation, is viewed as a key factor associated with inflammation-related diseases (12). At the site of inflammation, ROS-induced oxidant stress (OS) is involved in irreversible injury of cells or tissues through DNA damage or peroxidation (13), causing pathological changes which could adversely impact the reproductive system, nervous system, respiratory system, etc. (14-16). Regarding to reproductive diseases, it has been confirmed that ROS is excessively accumulated in ovarian tissues during inflammation-mediated ovarian diseases and contributes to disease progression (17, 18). Meanwhile, there was report suggested that ovarian inflammation triggered by pathogenic $\mathrm{T}$ cells specific recognizing certain ZP3 epitopes maybe accompanied by ROS accumulation (19). Nevertheless, this hypothesis has not yet been evaluated in fertility studies and the link between OS and ovarian pathology remains elusive.

Selenium (Se) is considered as an essential trace mineral, which plays an important role in the health of animals and humans at the cell and organism levels (20). The biological functions of Se are mainly performed by selenoproteins, and related functions are conserved among mammal species $(21,22)$. As the main component of selenoprotein, Se can exert its catalytic and anti-oxidant functions by stabilizing structure and enzymatic activities, as well as regulate physiological processes such as DNA synthesis, clearance of harmful peroxides and redox signal control $(20,23)$, whereas suitable low-concentration Se can promotes activation of selenoproteins and suppress ROS production by trapping electrons released during oxidative phosphorylation as an anti-oxidative effect (24). Inorganic (selenates and selenites) and organic (selenomethionine and selenocysteine) Se are two forms of Se in the dietary profile for supplementation though oral and injection $(25,26)$. Generally, organic Se is preferred due to higher absorption efficiency and metabolic stability whereas the blood Se concentration can be boosted quickly after supplementation, leading to the accumulation in the systemic circulation. However, chronic Se poisoning occurs after long-term administration $(26,27)$. Conversely, although inorganic Se demonstrates lower utilization efficiency after administration, the toxicity of inorganic Se requires high-dose drug or high local concentration
$(26,28)$, which makes inorganic Se as favorable and injectable supplements for applying in the critical periods in the production cycle (28), or cancer therapeutics $(29,30)$.

Sodium selenite (SS) is the representative form of inorganic Se and there are conclusive evidences suggested that SS can prevent ROS accumulation in ovaries and prevent or reverse OS-induced ovarian dysfunction (31). The level of hormones and development of follicles were significantly improved after inhibition of ROS production using SS, whereas the capabilities of total antioxidant capacity (TAC) was also enhanced (32). More importantly, the similar phenomenon were observed from randomized double-blind placebo-controlled trials in pregnant women, and the SS-induced improvement of antioxidant capacity has been shown to be closely related to fertility $(33,34)$. Therefore, the connection among fertility, OS and follicle during the ZP3-administration period has investigated in present study. Here, the $\mathrm{mZP} 3$ protein vaccination was used to establish an AOD mouse model. The OS level, follicle number and apoptosis level were evaluated performed after periodic $\mathrm{mZP} 3$ immunizations to verify the contribution of OS to follicle depletion of ZP3-induced subfertility mice with or without concomitant sodium selenite (SS) treatment, and ultimately to reveal the relationship between OS and fertility.

\section{MATERIALS AND METHODS}

\section{Animals and Antigens}

Adult female $\mathrm{BALB} / \mathrm{c}$ mice (6-8 weeks old) were purchased from Dashuo Biotech (Chengdu, Sichuan, China) received sterile water and food under daily 12-h light/12-h dark cycle conditions. All animal experiments in this study were approved by the Committee on Ethical Use of Animals of Northwest A\&F University. Care of animals was conducted with strict adherence to the guidelines of Northwest A\&F University Institutional Committee and every effort was made to minimize animal suffering.

Recombinant mouse $\mathrm{ZP} 3$ protein fused to glutathione S-transferase (GST) tag was produced by using a constructed plasmid pGEX-4T-1-mZP3 with the prokaryotic expression system as previously reported (35). The control protein GST was obtained using the aforementioned backbone plasmid and expression system as well. Briefly, pGEX-4T-1 was transformed into BL21 (DE3) cells (TransGen Biotech, Beijing, China) then protein expression was induced by incubation of cells (after the $\mathrm{OD}_{600 \mathrm{~nm}}$ value of bacteria reached $0.6-0.8$ ) with $0.2 \mathrm{M}$ isopropyl- $\beta$-D-thiogalactoside (IPTG) at $28^{\circ} \mathrm{C}$ for 8 hours shaking culture $(180 \mathrm{rpm})$. Next, a supernatant containing GST protein was obtained after ultra-sonication of bacterial followed by a high-speed centrifugation $(12,000 \times g)$ at $4^{\circ} \mathrm{C}$. Purification of GST protein from the supernatant was conducted according to the manufacturer's instructions using BeyoGold ${ }^{\mathrm{TM}}$ GST-tag Purification Resin (Beyotime Biotechnology, Beijing, China). Protein concentrations were quantified using a BCA protein assay kit (Thermo Fisher Scientific; Waltham, Massachusetts, USA). 


\section{Animal Immunization and SS Treatment}

Seventy-five female BALB/c mice were permitted to acclimate to their new environment for 7 days without any treatment. Referring to the previous study (36), after acclimation the mice were administered by intramuscular injections $(0.1 \mathrm{mg} / \mathrm{kg})$ of estradiol benzoate (EB; Sigma-Aldrich, St. Louis, MO, USA) for 3 consecutive days to synchronize their estrus cycles, and the signs of a large number of keratinized epithelial cells and a small number of nucleated epithelial cells in the vaginal wash are defined as estrus period though smear observation. Next, mice were randomly divided into three groups $(n=25)$ : (a) mZP3+SS; (b) mZP3+Saline; and (c) GST+Saline (negative control group). For mice receiving the immunogen $\mathrm{mZP} 3$ or GST: $100 \mu \mathrm{g}(1 \mathrm{mg} / \mathrm{mL})$ of antigen was mixed with Imject $^{\mathrm{TM}}$ Alum Adjuvant (Thermo Fisher Scientific) at a volume ratio of $1: 1$, and three rounds of immunization schedule are performed by subcutaneous injection at 2-week intervals. The drug SS (SigmaAldrich) was dissolved in saline with indicated concentration (0.5 $\mathrm{mg} / \mathrm{kg}$ ) after referring to the previous studies $(31,37)$, then 100 $\mu \mathrm{L}$ of SS or the same volume of saline as the negative control were administered to mice via intraperitoneal injection once daily throughout the entire immunization program before the mating procedure. Finally, ten mice in each group were selected randomly for assessments of litter size followed by ovarian histological analysis, while the remaining fifteen mice in each group were used solely for ovarian tissue-related tests.

\section{Animal Mating and Litter Size Statistics}

On day 36 after first administration, ten mice were coupled with normal males of similar age (one male for each female) with rotation of male mice conducted among the different cages daily. While females with successful mating were confirmed by monitoring of vaginal suppositories daily at 8:00 am during the mating period. Males were removed after coupling for 7 days. All males used in these experiments were previously confirmed to be fertile through mating with untreated females. The number of pups produced by each female were counted and compared with that of controls by 21-27 days after mating procedure.

\section{Sera Collection and Determination of the Antibody Levels}

Serum samples were collected from mice on the day of mating then were stored at $-80^{\circ} \mathrm{C}$ until use. The level of IgG in serum was determined by indirect enzyme-linked immunosorbent assay (iELISA). Briefly, 96-well plates (Nunc, Thermo Fisher Scientific) were coated with purified mZP3 or GST (400 ng/well) and incubated at $4{ }^{\circ} \mathrm{C}$ overnight. Next, solutions in wells were replaced with blocking buffer (PBS solution containing 5\% skimmed milk) then plates were incubated at room temperature (RT) for 1 hour. After washing wells once with PBS-T (PBS buffer containing $0.05 \%$ Tween 20 ), $100 \mu \mathrm{L}$ of serial 2-fold dilutions of serum in blocking buffer (from 1:100 to 1:1638400) were added to triplicate wells followed by 1 hour incubation of plates at RT. Wells were then washed three times with PBS-T then $100 \mu \mathrm{L}(0.2 \mu \mathrm{g} / \mathrm{mL})$ of HRP-conjugated goat anti-mouse IgG (TransGen Biotech) diluted in blocking buffer was added per well. Next, binding between the secondary antibody and primary antibody was allowed to proceed for 1 hour at RT. After liquid was removed from wells, wells were washed three times with PBS-T then 100 $\mu \mathrm{L} 3,3^{\prime}, 5,5^{\prime}$-tetramethylbenzidine (TMB; TransGen Biotech) was added per well for $10 \mathrm{~min}$ to trigger colorimetric reaction. Finally, $\mathrm{OD}_{450 \mathrm{~nm}}$ values were read using an Epoch-BioTek Microplate Reader after the reaction was terminated by adding of $3 \mathrm{M} \mathrm{H}_{2} \mathrm{SO}_{4}$ ( $50 \mu \mathrm{L} /$ well). Determination of the antibody titer for each mouse was conducted based on whether average $\mathrm{OD}_{450 \mathrm{~nm}}$ values of a given serum dilutions in triplicate (presented as the mean value plus three standard deviations) was greater than the mean value for the corresponding dilution of negative serum.

\section{Ovarian Collection and Histological Analysis}

Statistical analysis of numbers of pups in each group was conducted on day 70 after the first immunization, then $10 \%$ chloral hydrate (Sigma-Aldrich) $(10 \mathrm{mg} / \mathrm{kg})$ was injected intraperitoneally into each mouse to anesthetize the animal then ovaries were removed for histological preparation and analysis of ovarian tissue section. Briefly, ovaries of ten mice in each group were fixed immediately by immersion into $4 \%$ paraformaldehyde (Beyotime Biotechnology) after collection, fixed tissues were maintained at $4{ }^{\circ} \mathrm{C}$ for at least 48 hours then embedded in paraffin and sliced into $5 \mu \mathrm{m}$ section, followed by hematoxylin and eosin (HE) staining steps with Hematoxylin and Eosin (HE) Staining Kit (Beyotime Biotechnology) according to the manufacturer's instructions. Ovaries tissues with HE staining were examined according to the method of Myers et al., to identify the mouse follicles at different developmental stages and accurately estimate the number by the number of granulosa cells, not morphology. Primary follicle is totally surrounded by a single layer of cubic granular cells, while mature follicles is surrounded by a part of cumulus cells (38).

\section{Evaluation of ROS Levels in Mouse Ovarian Cells}

To assess ROS levels in mouse ovarian cells, a fluorescence probebased method was used. First, five randomly selected mice in each group were euthanized to collect ovaries using the same method as mentioned above. Next, tissues were pulverized to generate single-celled suspensions by pressing the tissues into a 200-mesh sterile copper screen using a glass rod. Resulting cell suspensions were centrifuged at $300 \times g$ for $10 \mathrm{~min}$ at $4^{\circ} \mathrm{C}$ then cell pellets were washed twice with Dulbecco's Modified Eagle's Medium (DMEM, Gibco, Carlsbad, CA, USA). Detection of ROS in ovarian cells was performed using a Reactive Oxygen Species Assay Kit (Beyotime Biotechnology) according to the manufacturer's instructions.

To further assess levels of OS-associated indicators in ovarian tissue, another five mice were randomly selected from each group for ovaries collection as well. Next, tissues were homogenized on ice at 1:10 $(\mathrm{w} / \mathrm{v})$ in saline $(P H=7.2)$ using a MagNA Lyser Tissue Homogenizer (Roche, Basel, Switzerland) then supernatants were obtained by centrifugation of homogenized specimens at $13,000 \times g$ for $20 \mathrm{~min}$ at $4^{\circ} \mathrm{C}$. Ovarian tissue supernatants were next evaluated for ROS and malondialdehyde (MDA) levels as well as total antioxidant capacity (T-AOC) using Mouse 
Reactive Oxygen Species ELISA Kit (MLBio, Shanghai, China), Malondialdehyde (MDA) Colorimetric Assay Kit (TBA method, Elabscience, Wuhan, China) and Total Antioxidant Capacity Colorimetric Assay Kit (Elabscience), respectively.

\section{Analysis of Mitochondrial Apoptosis-Related Proteins}

Ovaries from the remaining five mice in each group were obtained using the same methods as described above then were frozen in liquid nitrogen. All ovarian samples in each group were pooled together and ground into a fine powder using a mortar and pestle. Next, total protein was extracted using a Tissue or Cell Total Protein Extraction Kit (Sangon Biotech, Shanghai, China) according to the manufacturer's instructions. Finally, protein concentrations were determined using a BCA Protein Assay Kit (Thermo Fisher Scientific) according to the manufacturer's instructions.

For Western blot analysis, protein samples were first separated by sodium dodecyl sulfate-polyacrylamide gel electrophoresis (SDS-PAGE) then proteins were electro-transferred to the methanol-activated PVDF membranes. Next, membranes were blocked then incubated with primary and secondary antibodies as described above for the iELISA; primary antibodies (Beyotime Biotechnology) included anti-Bax, anti-Bcl-2, anti-caspase- 3 and anti-caspase- 9 that were each diluted 1:1,000. After membranes were washed, they were developed based on a colorimetric reaction with ECL Western Blot Substrate (Solarbio) then developed blots were viewed using a ChemiDoc MP Image System (Bio-Rad). Differences in protein expression levels among different groups were analyzed using Image J software (Image J2x, Rawak Software Inc., Stuttgart, Germany).

\section{Statistical Analysis}

All data are presented as the mean \pm SD. A scatter diagram of antibody levels, litter sizes and correlations between litter size, as well as antibody titer or number of follicles were determined using nonparametric correlation (Spearman's correlation). Determinations of statistical significance of line charts and histograms associated data within each group were all performed using GraphPad Prism version 7.0 (GraphPad Software, San Diego, CA, USA) using either the Student's $t$-test for the comparison of two groups or one-way analysis of variance (ANOVA) for testing of more than two groups. A two-tailed $P$ value $<0.05$ is considered to be statistically significant.

\section{RESULTS}

\section{Fertility Assessment of mZP3-Immunized Mice After SS Treatment}

Statistical analysis of data obtained in this study revealed no differences in fertility rates among the three groups $\mathrm{mZP} 3+\mathrm{SS}$, mZP3+Saline and GST+Saline, which were 90\% (9/10), 70\% (7/10) and 100\% (10/10), respectively. However, values of average mean litter size per mouse in both $\mathrm{mZP} 3+\mathrm{SS}$ and $\mathrm{mZP} 3+$ Saline groups were significantly lower at $4.3(P<0.05)$ and $2.4(P<$ 0.01 ) than that of the GST+Saline negative group of 6.5 (Table 1 ). Notably, SS treatment of the mZP3+SS group led to higher
TABLE 1 | Fertility of mice from different groups.

\begin{tabular}{lcccccc}
\hline Antigen & Mouse $^{\text {a }}$ & $\begin{array}{c}\text { No. } \\
\text { fertile }\end{array}$ & $\begin{array}{c}\% \\
\text { Fertile }^{\text {b }}\end{array}$ & $\begin{array}{c}\text { Total }^{\mathbf{c}} \\
\text { pups }\end{array}$ & $\begin{array}{c}\text { Mean litter size } \\
\text { of mice }\end{array}$ & $\boldsymbol{P}^{\mathbf{e}}$ \\
\hline mZP3 + SS & 10 & 9 & 90 & 43 & $4.3 \pm 2.41^{\star}$ & 0.007 \\
mZP3 + Saline & 10 & 7 & 70 & 24 & $2.4 \pm 1.43^{\star \star}$ & 0.013 \\
GST + Saline & 10 & 10 & 100 & 65 & $6.5 \pm 0.98$ &
\end{tabular}

${ }^{a}$ Female BALB/C mice were immunized with $m Z P 3$ protein and control protein GST at 2 week intervals for three times, and treated with chemical drug SS and control saline daily though the whole immunization process before mating. All mice were subjected to mating on day 36 after the first immunization.

${ }^{b}$ Percentage of fertile mice were calculated by the fertile numbers divided by the total mated females in each group.

${ }^{c}$ Total number of pups of each group were counted 21 27 days after animals mating.

${ }^{d}$ Mean litter size of mice were calculated by the total pups divided by the total mated females in each group.

${ }^{e}$ Significant differences $\left({ }^{*} P<0.05\right.$ and $\left.{ }^{* *} P<0.01\right)$ are presented between immunized groups and negative controls (GST+Saline). Values are the means $\pm S D$.

average litter size without affecting the ZP3-induced antibody titers, which was $\sim 29 \%$ higher than that of the drug control group mZP3+Saline, indicating a key role of OS in ZP3-vaccinationinduced subfertility. These results suggest that mZP3-induced OS likely further caused subfertility on the basis of antibodymediated fertility effects.

Determination of antibody titer for each mouse in the different groups indicated that strong immune responses against mZP3 and GST were observed in all mice, including the infertile one (Figure 1A). GST group mice with anti-GST antibody titers that were equivalent to anti-mZP3 antibody titers of mZP3+SS and $\mathrm{mZP} 3+$ Saline groups gave birth to the greatest numbers of pups, with no correlation found between anti-GST antibody titers and litter size (Pearson $r=0.0603$ ). Moreover, no significant difference was observed in numbers of litters among individual mice in this group $(P=0.8687)$ (Figure 1B). Meanwhile, in the mZP3+SS group a negative correlation was found between antibody level and litter size that was significantly more negative (Pearson $r=-0.7851$ ) than that obtained for the Non-treated, mZP3-immunized group (Pearson $r=-0.6089$ ). Furthermore, the $P$ values of $\mathrm{mZP} 3+\mathrm{SS}(P=0.0071)$ and $\mathrm{mZP} 3$-immunized $(P=0.0617)$ groups both are $<0.05$, suggesting that the above correlation analysis is highly reliable (Figure 1B).

\section{SS-Administration Restored the OS Induced Follicle Depletion}

Notably, in this work a significant follicle number reduction, especially the mature one, were observed in ovaries of the mZP3+Saline group as compared to the negative control group. Specifically, mean numbers of primary and mature follicles dropped from 7.2 and 6.8, for the negative control group to 4.3 and 2.9 , respectively, for the mZP3+Saline group (Figure 2Ab). Interestingly, numbers of corpus luteum (CL) increased significantly in both fertile and infertile mice (Figure 2Aa), but did not contribute to fertility, indicating that the follicles were abnormally disappeared. In addition, although numbers of follicles differed significantly among individual mice, the total number of follicles in mZP3-immunized mice were 
A

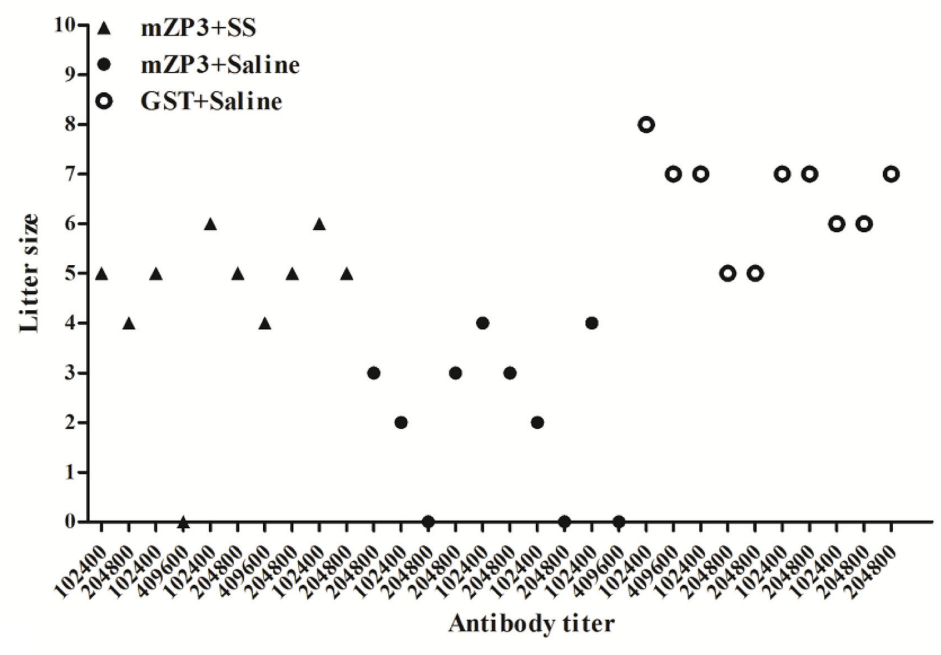

B
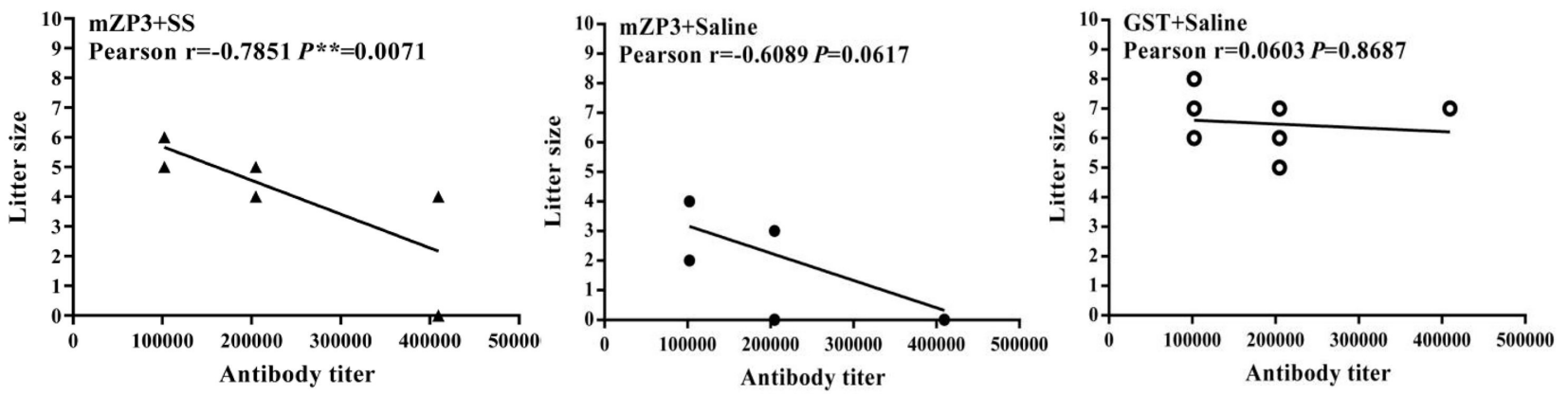

FIGURE 1 | ZP3-specific antibody shows a significant negative correlation with litter size. (A) Scatter diagram of antibody levels and litter size after mating. The antibody levels of each mouse were determined by serum serial 2-fold dilution from 1:100 to 1:819200 in an iELISA assay, and corresponds to their own litter size. (B) Correlation analysis between antibody titer and litter size. The Pearson coefficient $r$ value means the correlation between antibody titer and litter size, while $P$ value $<$ 0.05 indicates credibility of $r$ value.

significantly increased in mice that received SS treatment, while primary follicle numbers were comparable to numbers for the negative control group (Figure 2Ab). More importantly, the correlation analysis revealed that numbers of primary and mature follicles were both significantly positively correlated with litter size $(r=0.8683$ and $r=0.7894 ; r=0.9276$ and $r=$ 0.8830 ) (Figure 2Ba,b). Surprisingly, the credibility of correlation analysis in the SS-treated, mZP3-immunized group (Figure 2Ba) decreased from $P<0.001$ to $P<0.01$ as compared to that of the untreated mZP3-immunized group (Figure 2Bb). Meanwhile, although not significant, the correlation between primary and mature follicles with litter size also decreased from Pearson $r$ $=0.9276$ and Pearson $r=0.8830$, respectively (Figure 2Bb), in the mZP3+Saline group to Pearson $r=0.8683$ and Pearson $r=$ 0.7894 in mZP3 + SS group, respectively (Figure 2Ba).

\section{OS Is Associated With ZP3-Induced Subfertility}

Due to the abovementioned beneficial effects of SS treatment for improving fertility and follicle development, evaluations of ovarian OS levels were conducted concurrently to reveal the mechanism underlying development of subfertility in mZP3-immunized mice. As shown in Figure 3A, obvious OS accumulation in ovarian cells was observed in mZP3immunized mice. Subsequently, this accumulation was further confirmed by checking ovarian tissue homogenate ROS levels, and declared that the ROS level was elevated in ovarian tissue by about $84 \%$ as compared to that of the negative control group (Figure 3B). Additionally, MDA content associated with membrane damage also showed a significant increase (by about 58\%), as expected (Figure 3C). In addition, evaluation of ovarian T-AOC demonstrated a significant decrease also when abovementioned metabolic ROS homeostasis was disrupted, with consistent results observed here whereby T-AOC dropped by about 40\% (Figure 3D). Although mZP3-induced ovarian OS was not completely diminished by SS treatment, administration of SS during mZP3 immunization alleviated OS to some degree, such that levels of all OS-related indicators in $\mathrm{mZP} 3+\mathrm{SS}$ mice were more than $40 \%$ lower than corresponding indicators for mice immunized with $\mathrm{mZP} 3$ alone. 
A
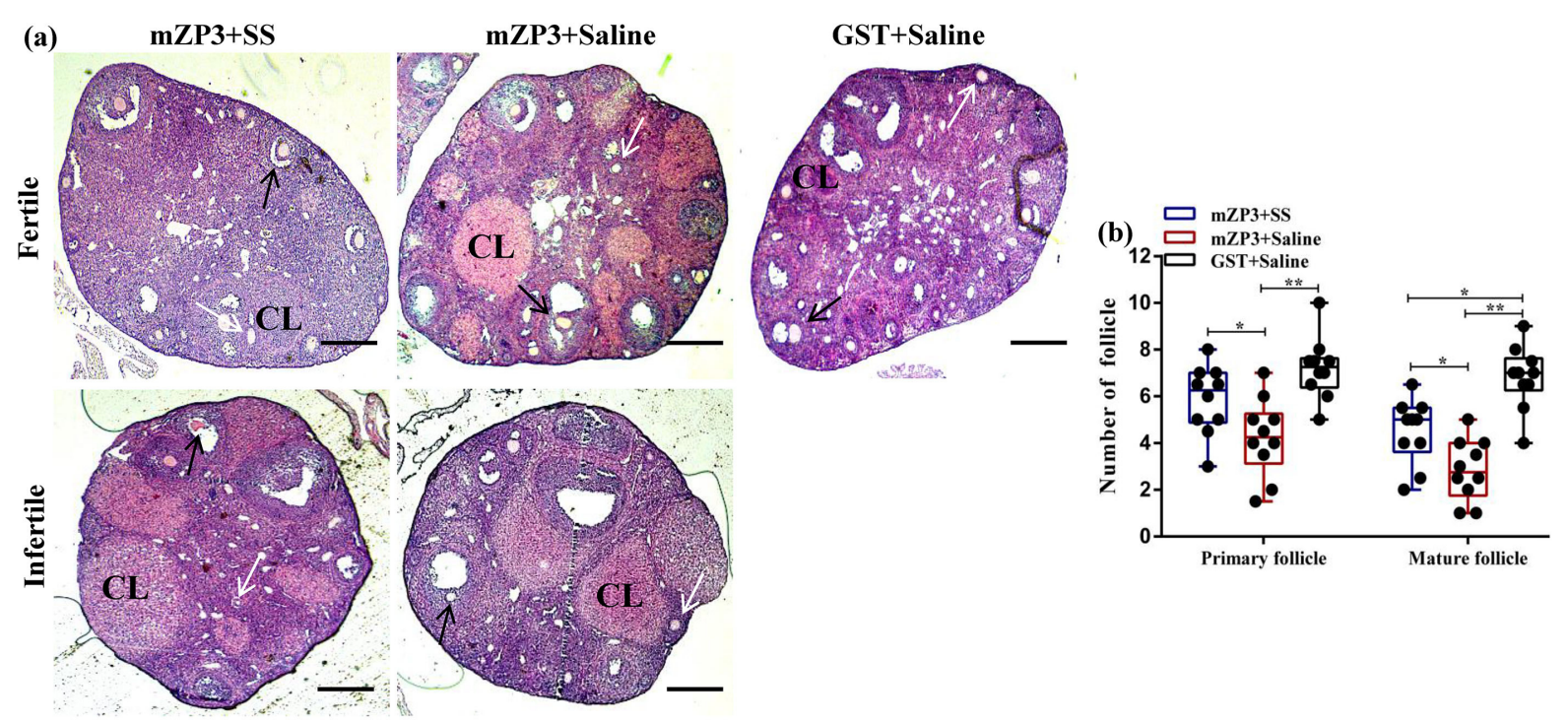

B
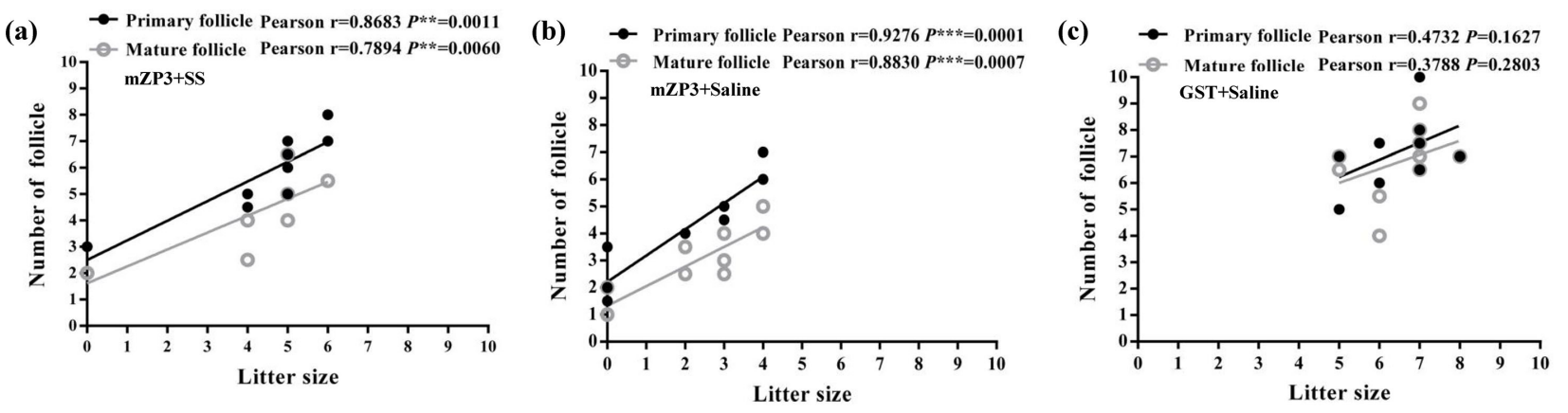

FIGURE 2 | Follicle depletion is associated with litter size decline in mZP3 immunized mice. (A) Histological analysis of ovaries of mZP3+SS, mZP3+Saline and GST+Saline immunized mice. Mice ovaries were collected on day 70 after the first immunization, followed by tissue sectioning and HE staining for primary and mature follicles count $(40 \times)(a)$, then performed statistical analysis among the indicated groups are marked as "*” or " "**", which denotes $P<0.05$ and $P<0.01$, respectively, and those with insignificant differences are not marked $(P>0.05)(b)$. CL: Corpus luteum. Black arrows indicate primary follicles; while arrows indicate mature follicles. (B) Correlation analysis between litter size and follicle number. The number of primary and mature follicles were counted based on the obtained ovarian sections above, and performed correlation analysis with litter size of mZP3+SS (a), mZP3+Saline (b) and GST+Saline (c) immunized mice. The Pearson coefficient $r$ value means the correlation between antibody titer and litter size, while $P$ value $<0.05$ indicates credibility of $r$ value.

\section{Vaccination-Induced OS Promotes Apoptosis of Ovarian Cells}

Considering that numbers of ovarian follicles and ROS homeostasis both declined in mZP3-immunized mice, the hypothesis that ovarian pathology leads to mitochondrial apoptosis and abnormal follicular development may be correct. To further test this hypothesis, activation of key apoptosis-related proteins caspase- 3 and caspase- 9 as well as changes in expression levels of pro-apoptotic protein Bax and anti-apoptotic protein Bcl-2 were assessed to determine the degree of cell apoptosis in the ovary. Toward this goal, Western blot results demonstrated that the first three aforementioned proteins were all up-regulated after mZP3 immunization, while expression of $\mathrm{Bcl}-2$ protein was significantly reduced (Figure 4A). Interestingly, the mZP3 effect for promoting apoptosis was significantly reversed by simultaneous treatment of SS. Specifically, relative levels of activated caspase-3 and caspase- 9 in the mZP3+SS group only increased by 17 and $12 \%$, respectively, as compared to corresponding levels in GST-immunized mice and mock-treated mice, while corresponding levels in $\mathrm{mZP} 3$-immunized mice were increased by $\sim 70 \%$ and $\sim 58 \%$, respectively (Figure $4 \mathrm{~B}$ ). Meanwhile, up-regulation of Bax protein expression in $\mathrm{mZP} 3$ immunized, SS-treated mice was reduced by $30 \%$ as compared to that of untreated mZP3-immunized mice. In addition, it is worth noting that $\mathrm{SS}$ treatment not only restored $\mathrm{Bcl}-2$ expression levels in $\mathrm{mZP} 3$-immunized mice, but led to elevated levels as compared to those of untreated, but immunized mice. In the final analysis, levels of anti-apoptosis indicators in ovarian cells of mZP3-immunized mice treated with the OS-specific drug SS were all significantly greater $(P<0.05)$ than corresponding levels observed for untreated, mZP3-immunized mice based on the detection of apoptosis-associated proteins (Figure 4B). 


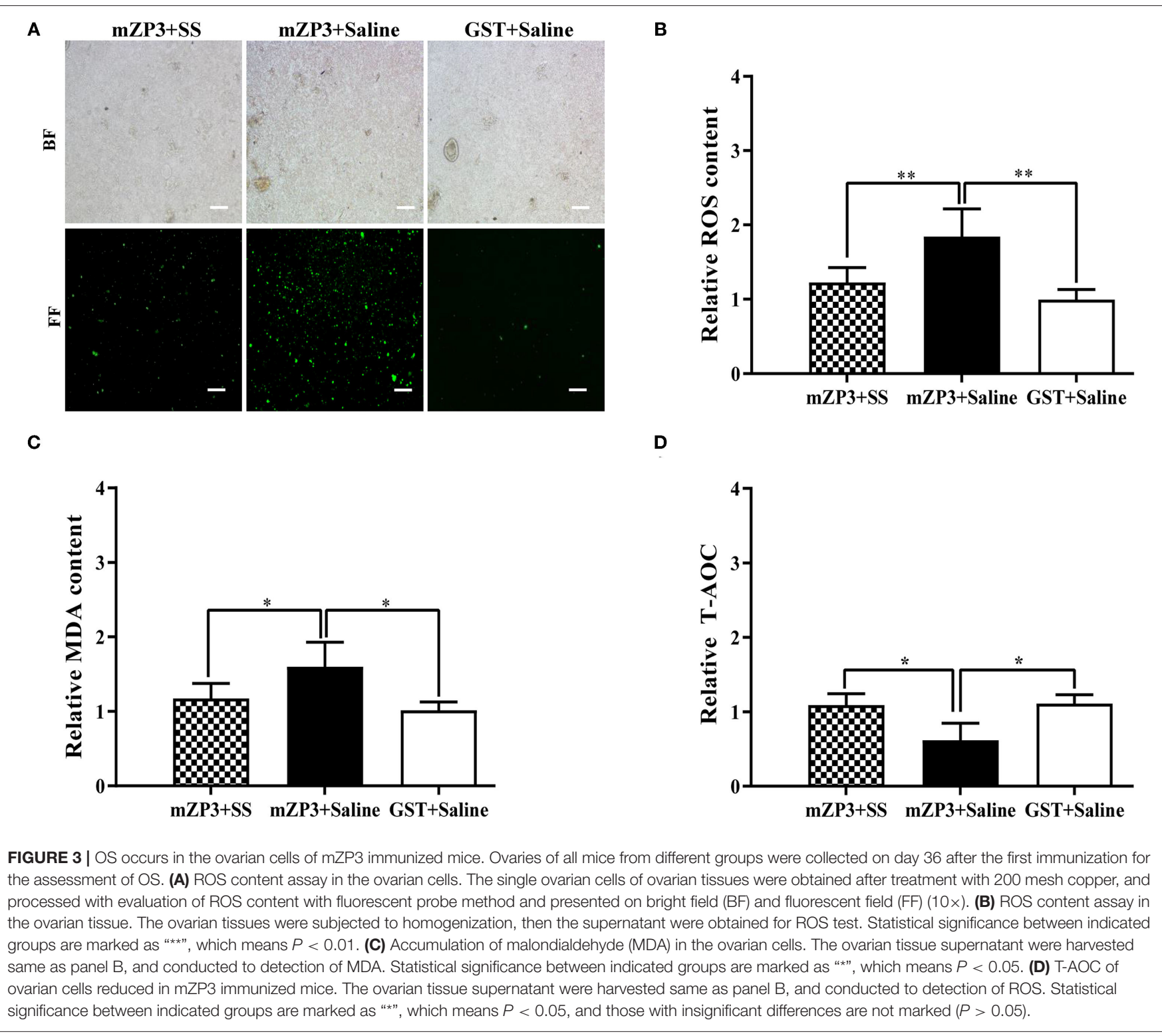

\section{DISCUSSION}

By targeting to block sperm-preferred receptors or enhancing antibody-mediated steric hindrance of sperm, high-titer antibodies produced against ZP3 have been shown to inhibit fertilization, which is a key mechanism of infertility $(2,3,39)$. Moreover, the presence of auto-antibodies to $\mathrm{ZP}$ in sera of infertile women has also been shown to be closely related to infertility (40), and negatively correlated with fertilization in vitro and fertility in vivo $(6,41)$. Although no evidence exists to declared that the presence of $\mathrm{ZP} 3$ antibodies affects the formation of the follicular ZP structure, and once this structure was formed thus ZP3 antibodies had minimum effect on development of the fertilized egg after implantation (42), the fundamental role of ZP3-specific antibodies within the vaccination induced infertility is confirmed by present study. Our data suggested that mice immunized with recombinant $\mathrm{mZP} 3$ protein containing intact pathogenic epitopes did indeed exhibit ovarian pathological damage, whereby mZP3-immunized mice had higher titers of ZP3-specific antibodies gave birth to fewer pups. In addition, correlation analysis revealed a significant negative correlation between antibody level and litter size, indicating the leading role of ZP3-specific antibody-mediated subfertility. More importantly, once the progressive declining of follicles was interrupted by SS treatment, the coefficient $r$ value representing the correlation between antibody and litter size has changed from 0.0617 to 0.0071 , of which further confirm the conclusion that antibodies dominate infertility.

Here, results obtained via examination of ovarian tissue sections of fertile and infertile mice suggested that numbers of follicles, especially mature follicles, was significantly reduced and associated with fewer litters. Furthermore, highly significant 
A

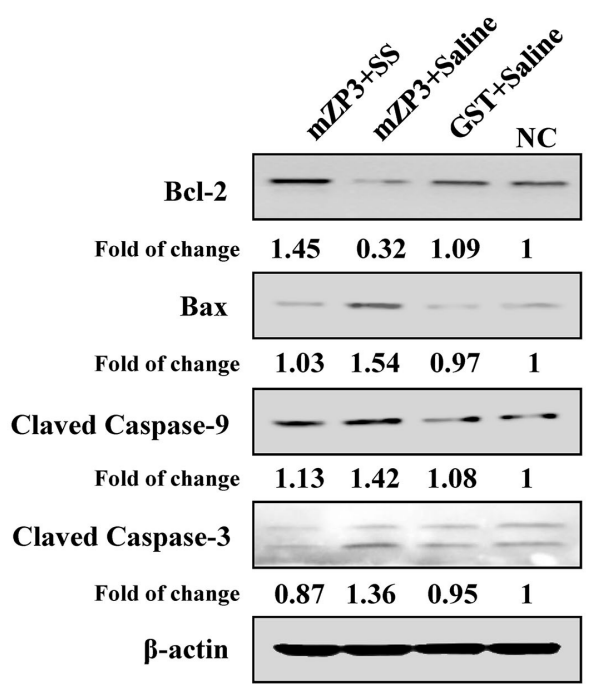

B
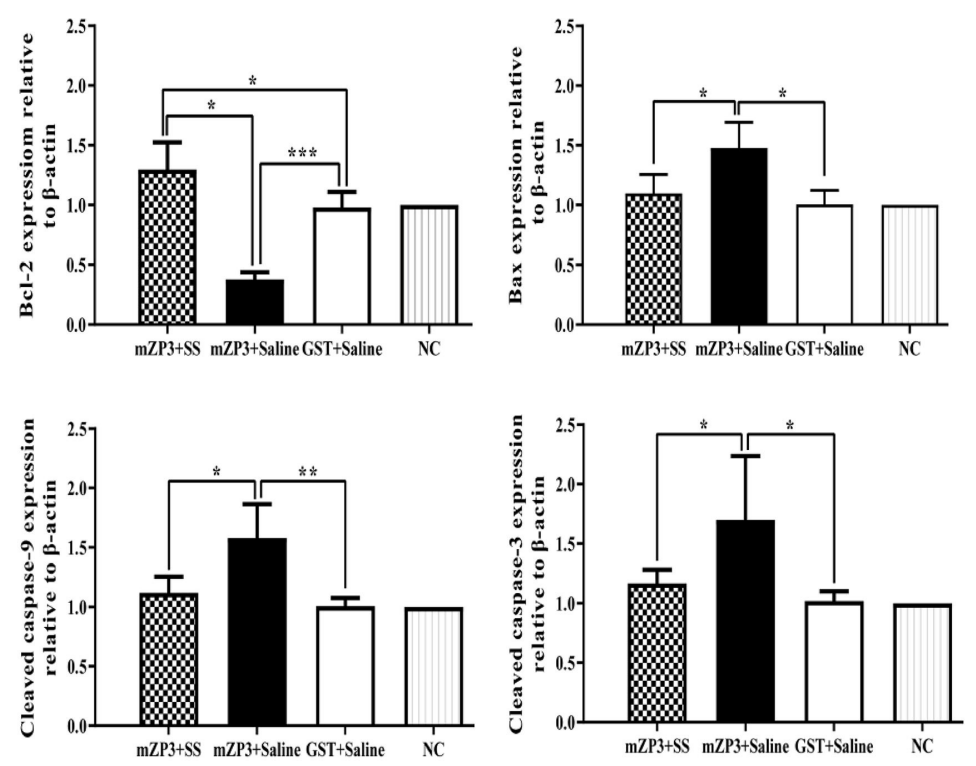

FIGURE 4 | The mZP3 vaccination causes apoptosis of ovarian cells in mice. (A) Apoptosis-related proteins increases in ovarian cells of mZP3 immunized mice. Ovaries were collected on day 36 after the first immunization, then performed the extraction of total protein and Western blot for detection of apoptosis-related proteins. Mock-immunized and treated mice were used as the negative control (NC). (B) Statistical analysis of Bax, Bcl-2, cleaved-caspase-9 and cleaved-caspase-3

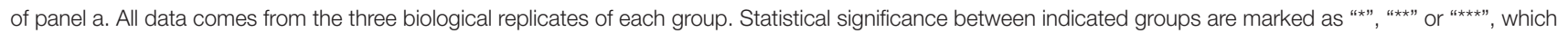
denotes $P<0.05, P<0.01$ and $P<0.001$, respectively, and those with insignificant differences are not marked $(P>0.05)$.

differences were observed in ovarian follicles numbers of mice belonging to different experimental groups, in spite of the fact that mice with near equivalent antibody titer to their mZP3. Although the specific underlying mechanism for this effect is unknown, these results strongly indicated closely relationship between ZP3 administration and follicle number decline of immunized mice as reported previously (8). Nevertheless, it is speculated that AOD caused by $\mathrm{T}$ cells recognizing a pathogenic $\mathrm{T}$ cell epitope within the ZP3 protein accelerates depletion of follicles via an unknown process $(4,43)$. Moreover, the CL is the collapsed structure of the follicular wall remaining in the ovary after ovulation, is closely related to the ovulation cycle of the female individual $(44,45)$, and both increased significantly in the SS-treated and ZP3-immunized mice without SS treatment, especially the infertile one. However, neither the number of follicles nor the litter size was improved in those mice with increased CL and showed no relationship, indicating an unknown factor of follicles disappearance caused subfertility. Meanwhile, considering ROS resulted inflammation and tissue injury $(13,19)$, the AOD induced by ZP3 immunization has been shown to possess characteristics of inflammation, follicle dysplasia and follicle failure, etc. (4, 5, 46), prompting us to further assess ovarian ROS levels. As expected, ROS accumulation in ovarian cells and whole tissues reached high levels, while MDA and T-AOC levels were increased and decreased, respectively, suggesting a serious OS and potential mitochondrial dysfunction in ovarian cells of ZP3-immunized mice, and the resulting side effects maybe also associated with the inflammation in the AOD model $(11,18,19)$. Moreover, there are conclusive evidence has demonstrated that mitochondrial OS is a key activator of apoptosis-induced follicles depletion (47-50). Therefore, evaluation of caspase, antiapoptotic and pro-apoptotic protein levels was further examined and it appeared that mitochondrial apoptotic pathway was involved in the progression of AOD, with apoptosis-induced follicular damage found to directly cause ovarian inflammationassociated reduction of follicle number. Meanwhile, abnormal mitochondria metabolism caused OS is probably involved in the apoptosis of ovarian cells and thus affect the development of follicles, which may provide an interpretation for the inconsistency of causality among increased CL and follicular reduction or litter decline after ZP3 vaccination.

Previously study demonstrated that SS treatment was shown to significantly promote development of follicles, oocytes and embryos in vitro by reducing ROS and increasing T-AOC levels (32). Meanwhile, SS treatment has also been shown to significantly increase OS levels in ovaries and uterus by inhibiting expression of activated caspase protein (31). Here, SS treatment of mZP3-immunized mice alleviated ovarian dysfunction by enhancing anti-OS ability and decreasing apoptosis levels. Statistical analysis of ROS and MDA results indicated levels of both were significantly decreased and that T-AOC level was significantly increased as compared to corresponding levels in untreated, mZP3-immunized mice. Therefore, our results suggested that OS depleted follicles via apoptosis, as reported previously (51-53). Moreover, as is a 
lysosome-mediated degradation process for Non-essential or damaged cell components, autophagy may participates in the above abovementioned OS-induced mitochondrial dysfunction of mZP3-immunized mice ovaries, but more investigations is needed (54). It is worth noting that our results demonstrated that SS co-administration with mZP3 immunization not only restored the number of follicles, but also significantly promoted increased litter sizes for almost all SS-treated, mZP3-immunized mice $(P$ $<0.05)$; by contrast, an inverse correlation was found between litter size and anti-mZP3 antibody titers $(P<0.01)$. Additional research by Said et al., has also found that SS-treatment maintained serum levels of follicle-stimulating hormone (FSH) and estradiol (E2) to prevent permanent follicle loss and further stimulate nuclear antigen (PCNA)-mediated follicular proliferation that ultimately improved ovarian function (31). Thus, these results indicate that the infertility mechanism caused by ZP3 vaccination induced ovarian dysfunction is extremely complicated, while the role of OS-related interlocking events during follicular development also should not be underestimated. In fact, during later stages of follicular development, reduction of follicle numbers in $\mathrm{mZP} 3$-immunized mice is likely the fundamental cause of follicle exhaustion that is associated with infertility $(55,56)$.

\section{CONCLUSION}

Results of the present study reveal a vital role of OSinduced follicle loss for the first time in the ZP3-based immunocontraception model. Specifically, the normal metabolism of mitochondria in ovarian cells was disrupt by the OS caused by inflammation, whereby the accumulation of ROS triggers the process of apoptosis and follicles depletion, and ultimately leading to the fertility decline of fertility. However, co-administration of SS with ZP3 immunization not only reversed the above harmful process, but also improved follicle number and litter size either effectively. Taken together, these results indicated that OS and related side effects are responsible

\section{REFERENCES}

1. Zhang D, Zhu L, Liu Z, Ren X, Yang X, Li D, et al. A novel mutation in ZP3 causes empty follicle syndrome and abnormal zona pellucida formation. J Assist Reprod Genet. (2021) 38:251-9. doi: 10.1007/s10815-02001995-0

2. Gupta SK, Srinivasan VA, Suman P, Rajan S, Nagendrakumar SB, Gupta N, et al. Contraceptive vaccines based on the zona pellucida glycoproteins for dogs and other wildlife population management. Am J Reprod Immunol. (2011) 66:51-62. doi: 10.1111/j.1600-0897.2011.01004.x

3. Shrestha A, Wadhwa N, Gupta SK. Evaluation of recombinant fusion protein comprising dog zona pellucida glycoprotein-3 and Izumo and individual fragments as immunogens for contraception. Vaccine. (2014) 32:564-71. doi: 10.1016/j.vaccine.2013.11.078

4. Li J, Jin H, Zhang F, Du X, Zhao G, Yu Y, et al. Treatment of autoimmune ovarian disease by co-administration with mouse zona pellucida protein 3 and DNA vaccine through induction of adaptive regulatory T cells. J Gene Med. (2008) 10:810-20. doi: 10.1002/jgm.1200

5. Tung K, Agersborg S, Bagavant H, Garza K, Wei K. Autoimmune ovarian disease induced by immunization with zona pellucida for observed follicle loss and associated with closely subfertility. Thus, infertility model resulted from the dominant effect of ZP3-specific antibody-mediated infertility during early stages of immunization and a Non-dominant role of OS at later stages of follicle development together.

\section{DATA AVAILABILITY STATEMENT}

The original contributions presented in the study are included in the article/supplementary material, further inquiries can be directed to the corresponding author.

\section{ETHICS STATEMENT}

The animal study was reviewed and approved by Committee on Ethical Use of Animals of Northwest A\&F University.

\section{AUTHOR CONTRIBUTIONS}

YN and E-MZ: conceived and designed the experiments. BZ and GQ: performed the experiments. BZ and YN: analyzed the data. BZ: writing-original draft preparation. YN: writing-reviewing and editing. All authors contributed to the article and approved the submitted version.

\section{FUNDING}

This work was supported by the grants from the National Natural Science Foundation of China awarded to YN (No. 31672534).

\section{ACKNOWLEDGMENTS}

We thank Professor Fuchun Zhang (College of Life Science and Technology, Xinjiang University), for generously providing the plasmids and draft revision support.
(ZP3) peptide. Curr Protoc Immunol. (2002) Chapter 15:Unit 15.17. doi: 10.1002/0471142735.im1517s49

6. Lou Y, Ang J, Thai H, McElveen F, Tung KS. A zona pellucida 3 peptide vaccine induces antibodies and reversible infertility without ovarian pathology. $J$ Immunol. (1995) 155:2715-20.

7. Liu D, Tu X, Huang C, Yuan Y, Wang Y, Liu X, et al. Adoptive transfers of $\mathrm{CD} 4(+) \mathrm{CD} 25(+)$ Tregs partially alleviate mouse premature ovarian insufficiency. Mol Reprod Dev. (2020) 87:887-98. doi: 10.1002/mrd. 23404

8. O'Leary S, Lloyd ML, Shellam GR, Robertson SA. Immunization with recombinant murine cytomegalovirus expressing murine zona pellucida 3 causes permanent infertility in $\mathrm{BALB} / \mathrm{c}$ mice due to follicle depletion and ovulation failure. Biol Reprod. (2008) 79:849-60. doi: 10.1095/biolreprod.108.067884

9. Chen T, Bian Y, Liu X, Zhao S, Wu K, Yan L, et al. A Recurrent missense mutation in ZP3 causes empty follicle syndrome and female infertility. Am J Hum Genet. (2017) 101:459-65. doi: 10.1016/j.ajhg.2017.08.001

10. Joone CJ, Schulman ML, Bertschinger HJ. Ovarian dysfunction associated with zona pellucida-based immunocontraceptive vaccines. Theriogenology. (2017) 89:329-37. doi: 10.1016/j.theriogenology.2016.09.018 
11. Turrens JF. Mitochondrial formation of reactive oxygen species. J Physiol. (2003) 552:335-44. doi: 10.1113/jphysiol.2003.049478

12. Griffith B, Pendyala S, Hecker L, Lee PJ, Natarajan V, Thannickal VJ. NOX enzymes and pulmonary disease. Antioxid Redox Signal. (2009) 11:250516. doi: 10.1089 /ars.2009.2599

13. Mittal M, Siddiqui MR, Tran K, Reddy SP, Malik AB. Reactive oxygen species in inflammation and tissue injury. Antioxid Redox Signal. (2014) 20:112667. doi: 10.1089/ars.2012.5149

14. Kim SM, Hwang KA, Choi KC. Potential roles of reactive oxygen species derived from chemical substances involved in cancer development in the female reproductive system. BMB Rep. (2018) 51:557-62. doi: 10.5483/BMBRep.2018.51.11.056

15. Kawamoto K, Sato I, Yoshida M, Tsuda S. Air purifiers that diffuse reactive oxygen species potentially cause DNA damage in the lung. J Toxicol Sci. (2010) 35:929-33. doi: $10.2131 /$ jts.35.929

16. Nissanka N, Moraes CT. Mitochondrial DNA damage and reactive oxygen species in neurodegenerative disease. FEBS Lett. (2018) 592:72842. doi: 10.1002/1873-3468.12956

17. Saed GM, Diamond MP, Fletcher NM. Updates of the role of oxidative stress in the pathogenesis of ovarian cancer. Gynecol Oncol. (2017) 145:595602. doi: 10.1016/j.ygyno.2017.02.033

18. Abizadeh M, Novin MG, Amidi F, Ziaei SA, Abdollahifar MA, Nazarian H. Potential of auraptene in improvement of oocyte maturation, fertilization rate, and inflammation in polycystic ovary syndrome mouse model. Reprod Sci. (2020) 27:1742-51. doi: 10.1007/s43032-020-00168-9

19. El-Kenawi A, Ruffell B. Inflammation, ROS, and Mutagenesis. Cancer Cell. (2017) 32:727-9. doi: 10.1016/i.ccell.2017.11.015

20. Rayman MP. The importance of selenium to human health. Lancet. (2000) 356:233-41. doi: 10.1016/S0140-6736(00)02490-9

21. Kryukov GV, Castellano S, Novoselov SV, Lobanov AV, Zehtab O, Guigo $\mathrm{R}$, et al. Characterization of mammalian selenoproteomes. Science. (2003) 300:1439-43. doi: 10.1126/science.1083516

22. Labunskyy VM, Hatfield DL, Gladyshev VN. Selenoproteins: molecular pathways and physiological roles. Physiol Rev. (2014) 94:739-77. doi: 10.1152/physrev.00039.2013

23. Duntas LH. Selenium and inflammation: underlying anti-inflammatory mechanisms. Horm Metab Res. (2009) 41:443-7. doi: 10.1055/s-0029-1220724

24. Colle D, Santos DB, de Souza V, Lopes MW, Leal RB, de Souza Brocardo P, et al. Sodium selenite protects from 3-nitropropionic acid-induced oxidative stress in cultured primary cortical neurons. Mol Biol Rep. (2019) 46:75162. doi: 10.1007/s11033-018-4531-y

25. Shchelkunov LF, Dudkin MS, Golubkina NA, Gins VK, Kononkov PF. Selenium and its role in nutrition. Gig Sanit. (2000) 32-5. https://www.ncbi. nlm.nih.gov/pubmed/11030107

26. Hadrup N, Ravn-Haren G. Absorption, distribution, metabolism and excretion (ADME) of oral selenium from organic and inorganic sources: a review. J Trace Elem Med Biol. (2021) 67:126801-12. doi: 10.1016/j.jtemb.2021.126801

27. Steen A, Strom T, Bernhoft A. Organic selenium supplementation increased selenium concentrations in ewe and newborn lamb blood and in slaughter lamb meat compared to inorganic selenium supplementation. Acta Vet Scand. (2008) 50:7-12. doi: 10.1186/1751-0147-50-7

28. Ferreira GM, Petzer IM. Injectable organic and inorganic selenium in dairy cows-Effects on milk, blood and somatic cell count levels. Onderstepoort J Vet Res. (2019) 86:e1-8. doi: 10.4102/ojvr.v86i1.1664

29. Gandin V, Khalkar P, Braude J, Fernandes AP. Organic selenium compounds as potential chemotherapeutic agents for improved cancer treatment. Free Radic Biol Med. (2018) 127:80-97. doi: 10.1016/j.freeradbiomed.2018. 05.001

30. Fernandes AP, Gandin V. Selenium compounds as therapeutic agents in cancer. Biochim Biophys Acta. (2015) 1850:164260. doi: 10.1016/j.bbagen.2014.10.008

31. Said RS, Badr AM, Nada AS, El-Demerdash E. Sodium selenite treatment restores long-lasting ovarian damage induced by irradiation in rats: impact on oxidative stress and apoptosis. Reprod Toxicol. (2014) 43:8593. doi: 10.1016/j.reprotox.2013.11.005

32. Abedelahi A, Salehnia M, Allameh AA, Davoodi D. Sodium selenite improves the in vitro follicular development by reducing the reactive oxygen species level and increasing the total antioxidant capacity and glutathione peroxide activity. Hum Reprod. (2010) 25:977-85. doi: 10.1093/humrep/deq002

33. Mesdaghinia E, Rahavi A, Bahmani F, Sharifi N, Asemi Z. Clinical and metabolic response to selenium supplementation in pregnant women at risk for intrauterine growth restriction: randomized, double-blind, placebo-controlled trial. Biol Trace Elem Res. (2017) 178:14-21. doi: 10.1007/s12011-016-0911-0

34. Bizerea TO, Dezsi SG, Marginean O, Stroescu R, Rogobete A, BizereaSpiridon $\mathrm{O}$, et al. The link between selenium, oxidative stress and pregnancy induced hypertensive disorders. Clin Lab. (2018) 64:1593610. doi: 10.7754/Clin.Lab.2018.180307

35. Li J, Jin H, Zhang A, Li Y, Wang B, Zhang F. Enhanced contraceptive response by co-immunization of DNA and protein vaccines encoding the mouse zona pellucida 3 with minimal oophoritis in mouse ovary. J Gene Med. (2007) 9:1095-103. doi: $10.1002 /$ jgm. 1069

36. Parandin R, Behnam-Rassouli M, Mahdavi-Shahri N. Effects of neonatal exposure to zearalenone on puberty timing, hypothalamic nuclei of AVPV and ARC, and reproductive functions in female mice. Reprod Sci. (2017) 24:1293-303. doi: 10.1177/1933719116683808

37. Yonemoto J, Satoh H, Himeno S, Suzuki T. Toxic effects of sodium selenite on pregnant mice and modification of the effects by vitamin $\mathrm{E}$ or reduced glutathione. Teratology. (1983) 28:333-40. doi: 10.1002/tera.1420280305

38. Myers M, Britt KL, Wreford NG, Ebling FJ, Kerr JB. Methods for quantifying follicular numbers within the mouse ovary. Reproduction. (2004) 127:56980. doi: 10.1530/rep.1.00095

39. Gupta N, Shrestha A, Panda AK, Gupta SK. Production of tagfree recombinant fusion protein encompassing promiscuous $\mathrm{T}$ cell epitope of tetanus toxoid and dog zona pellucida glycoprotein3 for contraceptive vaccine development. Mol Biotechnol. (2013) 54:853-62. doi: 10.1007/s12033-012-9634-4

40. Deroux A, Dumestre-Perard C, Dunand-Faure C, Bouillet L, Hoffmann P. Female infertility and serum auto-antibodies: a systematic review. Clin Rev Allergy Immunol. (2017) 53:78-86. doi: 10.1007/s12016-016-8586-Z

41. Yu MF, Fang WN, Xiong GF, Yang Y, Peng JP. Evidence for the inhibition of fertilization in vitro by anti-ZP3 antisera derived from DNA vaccine. Vaccine. (2011) 29:4933-9. doi: 10.1016/j.vaccine.2011.04.130

42. Dean J, East IJ. Effects of anti-zona pellucida monoclonal antibodies on fertilization and early development. Adv Exp Med Biol. (1986) 207:3753. doi: 10.1007/978-1-4613-2255-9_4

43. Bagavant H, Adams S, Terranova P, Chang A, Kraemer FW, Lou Y, et al. Autoimmune ovarian inflammation triggered by proinflammatory (Th1) T cells is compatible with normal ovarian function in mice. Biol Reprod. (1999) 61:635-42. doi: 10.1095/biolreprod61.3.635

44. Bonato DV, Ferreira EB, Gomes DN, Bonato FGC, Droher RG, Morotti F, et al Follicular dynamics, luteal characteristics, and progesterone concentrations in synchronized lactating Holstein cows with high and low antral follicle counts. Theriogenology. (2021) 179:223-9. doi: 10.1016/j.theriogenology.2021.12.006

45. Sievert LL. Physiology of the Graafian follicle and ovulation. Am J Hum Biol. (2004) 16:172-3. doi: 10.1002/ajhb.20004

46. Rhim SH, Millar SE, Robey F, Luo AM, Lou YH, Yule T, et al. Autoimmune disease of the ovary induced by a ZP3 peptide from the mouse zona pellucida. J Clin Invest. (1992) 89:28-35. doi: 10.1172/JCI115572

47. Lee JE, Park JH, Shin IC, Koh HC. Reactive oxygen species regulated mitochondria-mediated apoptosis in PC12 cells exposed to chlorpyrifos. Toxicol Appl Pharmacol. (2012) 263:148-62. doi: 10.1016/j.taap.2012. 06.005

48. Tammaro S, Simoniello P, Filosa S, Motta CM. Block of mitochondrial apoptotic pathways in lizard ovarian follicle cells as an adaptation to their nurse function. Cell Tissue Res. (2007) 327:625-35. doi: 10.1007/s00441-006-0256-7

49. Kleih M, Bopple K, Dong M, Gaissler A, Heine S, Olayioye MA, et al. Direct impact of cisplatin on mitochondria induces ROS production that dictates cell fate of ovarian cancer cells. Cell Death Dis. (2019) 10:85162. doi: 10.1038/s41419-019-2081-4

50. Peng $\mathrm{H}$, Zeng L, Zhu L, Luo S, Xu L, Zeng L, et al. Zuogui Pills inhibit mitochondria-dependent apoptosis of follicles in a rat model of premature ovarian failure. J Ethnopharmacol. (2019) 238:11185565. doi: $10.1016 /$ j.jep.2019.111855 
51. Wang S, Sun M, Yu L, Wang Y, Yao Y, Wang D. Niacin inhibits apoptosis and rescues premature ovarian failure. Cell Physiol Biochem. (2018) 50:206070. doi: 10.1159/000495051

52. Hayashi S, Nakamura T, Motooka Y, Ito F, Jiang L, Akatsuka S, et al. Novel ovarian endometriosis model causes infertility via iron-mediated oxidative stress in mice. Redox Biol. (2020) 37:101726-35. doi: 10.1016/j.redox.2020. 101726

53. Ghatebi M, Zavareh S, Lashkarbolouki T, Elahdadi Salmani M. Implications from early life stress on the development of mouse ovarian follicles: focus on oxidative stress. J Obstet Gynaecol Res. (2019) 45:1506-14. doi: 10.1111/jog. 14007

54. West RJ, Sweeney ST. Oxidative stress and autophagy: mediators of synapse growth? Autophagy. (2012) 8:284-5. doi: 10.4161/auto.8.2. 18981

55. Spears N, Lopes F, Stefansdottir A, Rossi V, De Felici M, Anderson RA, et al. Ovarian damage from chemotherapy and current approaches to its protection. Hum Reprod Update. (2019) 25:673-93. doi: 10.1093/humupd/ dmz027

56. Hao X, Anastacio A, Liu K, Rodriguez-Wallberg KA. Ovarian follicle depletion induced by chemotherapy and the investigational stages of potential fertility-protective treatments-a review.
Int
4720

Mol Sci.
(2019)
20:4720-46. doi:
$10.3390 /$ ijms 2019

Conflict of Interest: The authors declare that the research was conducted in the absence of any commercial or financial relationships that could be construed as a potential conflict of interest.

Publisher's Note: All claims expressed in this article are solely those of the authors and do not necessarily represent those of their affiliated organizations, or those of the publisher, the editors and the reviewers. Any product that may be evaluated in this article, or claim that may be made by its manufacturer, is not guaranteed or endorsed by the publisher.

Copyright (c) 2022 Zhang, Qu, Nan and Zhou. This is an open-access article distributed under the terms of the Creative Commons Attribution License (CC BY). The use, distribution or reproduction in other forums is permitted, provided the original author(s) and the copyright owner(s) are credited and that the original publication in this journal is cited, in accordance with accepted academic practice. No use, distribution or reproduction is permitted which does not comply with these terms. 